\title{
Sound Parameters for Expressing Geographic Distance in a Mobile Navigation Application
}

\author{
Mats Liljedahl \\ Interactive Institute, Sonic Studio \\ Acusticum 4 \\ SE-941 28 Piteå, Sweden \\ +467054058 88 \\ mats.liljedahl@tii.se
}

\author{
Stefan Lindberg \\ Interactive Institute, Sonic Studio \\ Acusticum 4 \\ SE-941 28 Piteå, Sweden \\ +46706644996 \\ stefan.lindberg@tii.se
}

\begin{abstract}
This paper presents work on finding acoustic parameters suitable to convey a sense of difference in geographic distance through the concepts of "near", "middle" and "far". The context for use is a mobile application for navigation services. A set of acoustic parameters was selected based on how sound naturally travels through and is dispersed by the atmosphere. One parameter without direct acoustic connection to distance was also selected. Previous works corroborate the choice of parameters in the context of the project. Results show that modulating multiple parameters simultaneously to express distance gives a more robust experience of difference in distance compared to modulating single parameters. The ecological parameters low-pass filter and reverb gave the test's subjects the most reliable and consistent experience of difference in distance. Modulating the parameter pitch alone was seen to be an unreliable method. Combining the pitch parameter with the reverb parameter gave more robust results.
\end{abstract}

\section{Categories and Subject Descriptors}

H.5.2 User Interfaces. Auditory (non-speech) feedback.

\section{General Terms}

Human Factors.

\section{Keywords}

Sonification, sound interaction design, acoustic parameters, geographic distance, mobile, navigation, auditory feedback.

\section{INTRODUCTION}

GPS-based navigation and travel services are becoming more and more common. These services are available in a growing number of devices, from dedicated navigator devices to multifunction mobile devices such as smartphones. Judging from the number of published apps offering navigational services for the iPhone and Android platforms, these apps represent popular and sought after services in peoples' lives. Many of these applications are based on

Permission to make digital or hard copies of all or part of this work for personal or classroom use is granted without fee provided that copies are not made or distributed for profit or commercial advantage and that copies bear this notice and the full citation on the first page. To copy otherwise, or republish, to post on servers or to redistribute to lists, requires prior specific permission and/or a fee.

Conference'10, Month 1-2, 2010, City, State, Country.

Copyright 2010 ACM 1-58113-000-0/00/0010 ..\$10.00 the traditional visual map metaphor, adding special signs denoting for example the user's current location or different types of points of interest. Over the last years, new types of views to support navigation and way finding have emerged that complement the traditional map. Several web-based services offer 360 degrees panorama views on street level of whole cities, see for example [9].

The traditional map is well suited to communicate geographic overview, directions to and relative distance between points [13]. New technologies such as GPS location and electronic compasses holds potential to create applications for navigation that complement the traditional, visual map by adding new qualities and dimensions to navigation tasks. Multi-modal interfaces using sound and/or vibration to give information about presence, direction, distance etc. to various types of points of interest to users is one track that researchers recently have started to explore. Such interfaces may not only be valuable for people that cannot use visual maps for various reasons. These interfaces also hold a potential to complement the traditional maps and add new values to the user experience.

The work reported here is part of a larger project working with the development of new, multimodal navigation services. The smartphones readily available today are equipped with sensors and other technologies that make it possible to create whole new types of navigation services. These new services can put other human abilities to play compared to the traditional navigation tools such as visually based maps, streetviews etc. In recent years, a series of projects have experimented with alternative ways to present geographical data and to create these new navigational services. Several projects have investigated vibration as cues for navigation, for example Pielot et al. [17]. The ability of sound to convey information about primarily directions to points of interest has also been investigated by for example Jones et al. [11], McGookin et al. [14] and Holland et al. [10].

One of the challenges for a navigation interface based on audio is to convey a sense of distance. Relatively few projects have looked at this problem and the field still lacks robust and well-grounded guidelines and practices on how to design auditory user interfaces to convey information about geographic distance.

In the work presented here, the expression of long-range distance, from tens of meters up to kilometers, through auditory cues is in focus. The aim is not to see how to give information about absolute distance, but instead find ways and acoustic properties well suited to convey a sense of the relative distance ranges "near", "middle" and "far". 


\section{BACKGROUND}

Devallez et al. looked at conveying depth information through sound in relatively short ranges [5]. Talbot and Cowan investigated how blind users perceive sound cues designed to express geographic distance [16]. Common to these and other work is that they utilize a mix of acoustic properties based on, what Talbot and Cowan call, "ecological cues". These cues are intensity or sound pressure, spectral filtering of sound with distance and the ratio of direct to reflected sound. Comparing the work by Devallez et al [5], Brungart [3] and Talbot and Cowan [16], it can be seen that using sound to express distance in the short range and the long range differ.

When sound waves travel through the atmosphere they are dispersed and affected in several ways. What we hear, the sound that reaches our ears will sound differently depending on how far from the sound source we are and thus how far the sound waves have traveled. The sound pressure decreases with distance. The farther away from a sound source we are, the weaker and less intense the sound [7]. The atmosphere attenuates sounds of different frequencies differently [7]. When sound waves travel through air, the friction between atoms absorbs higher frequencies more than lower frequencies. Therefore, the farther away from a sound source we are, the duller and more subdued the sound. The sound that we hear is also a mix of sound waves coming directly from the sound source and sound waves that have reflected on the ground and other surfaces before reaching our ears. The farther away from the sound source we are, the more reflected sound we will hear in relation to the sound coming directly from the sound source [7]. These three acoustic parameters affect the way sound works all the time around us. People without hearing impairments are used to listen to and to automatically, intuitively and subconsciously infer the distance to a sound source based on these physical properties of the sound [2][7][15].

Earlier experiments in the field have to a large extent focused on and tested single sound parameters and their ability to convey distance. Brungart [3] have in contrast made a series of experiments on distance perception through sound using discrete and combined sound parameters. Talbot and Cowan have also made late contributions to the understanding using combinations of parameters [16]. Both these bodies of work conclude that a combination of sound parameters gives a better or more accurate perception of distance compared to single-parameter conditions.

Non-ecological sound cues have, in contrast to the ecological, no connection to distance known to the user from everyday life. Instead the non-ecological parameters are definitions made by a designer of how to express distance. Pitch and beat rate are examples of non-ecological parameters used. As reported by Talbot and Cowan, early work on pitch to express distance gives inconsistent results. Some subjects interpret higher pitch as nearer and lower pitch as farther away, other subjects do the opposite interpretation. Talbot and Cowan found consistency in judgment of beat rate: higher beat rate (faster tempo) is closer.

Talbot and Cowan worked in a field similar to that of interest here, namely navigation services in mobile devices. One important difference is that their work is aimed at and based on visually impaired users whereas the work reported here is interested in and based on how non-visually impaired users perceive distance through auditory cues. As Talbot and Cowan points out, the perceptual abilities between the two groups are different. The motivation for this project is that there are still no clear answers to the question how distance can be conveyed through sound in the specific context of a mobile application for navigation for sighted users. It is therefore not evident that what works for the visually impaired does also work for the normally sighted.

The test described here was designed to assess how well a set of discrete sound parameters and combinations of those could be utilized to convey a sense of difference in the three geographic distances "near", "middle" and "far" to sighted users. The results from the test will be used directly in a project designing a soundbased navigation service for mobile devices. Even though these devices are increasingly powerful they do not have the computational power to compute the ecological sound parameters in real-time. Instead sound files with pre-rendered changes in acoustic parameters were and will be used. Because of this basic condition, the project is not interested in the judgment of absolute and continuous distances, but instead how to express the differences between the more arbitrary concepts of near, middle and far.

\subsection{Research Questions}

The project set out to answer two questions: First, how do subjects interpret changes in a set of acoustic parameters in terms of differences in geographic distance, and to what degree do they agree on an interpretation? Secondly, which acoustic parameter or combination of parameters do the subjects perceive as giving the most intuitive, direct and unambiguous sense of difference in geographic distance?

\section{SOUND PARAMETER SELECTION PROCESS}

When preparing the test, five acoustic parameters were initially appointed candidates to use, based on previous work: sound intensity (volume), pitch (frequency of tone), low-pass filter, reverb (reflections) and beat rate.

The mobile navigation application will be used at locations with varying geographic conditions. The way the application is designed, the number of sounds played will vary over time and with location. At some points no sounds will be played, at other points many sounds will be played in close succession. For this reason, the beat rate parameter is unsuitable for the context of the application and was therefore omitted from the test.

This leaves four parameters for the test: intensity, pitch, low-pass filter and reverb.

Table 1: Sound parameters with corresponding hypotheses about interpretation of distance

\begin{tabular}{|c|c|}
\hline Parameter & Hypothesis \\
\hline Intensity & $\begin{array}{l}\text { Lower intensity }=\text { greater distance } \\
\text { Higher intensity }=\text { smaller distance }\end{array}$ \\
\hline \multirow[t]{2}{*}{ Low-pass filter } & $\begin{array}{l}\text { Lower cutoff frequency = greater } \\
\text { distance }\end{array}$ \\
\hline & $\begin{array}{l}\text { Higher cutoff frequency }=\text { smaller } \\
\text { distance }\end{array}$ \\
\hline Reverb & More reverb $=$ greater distance \\
\hline & Less reverb $=$ smaller distance \\
\hline Pitch & $\begin{array}{l}\text { Lower pitch }=\text { greater distance } \\
\text { Higher pitch }=\text { smaller distance }\end{array}$ \\
\hline
\end{tabular}


A set of hypotheses was formulated for how test subjects would interpret sounds modified using these parameters with respect to geographic distance, see table 1. With the exception of the pitch parameter, the hypotheses were based on how sound behaves physically and how it is perceived in everyday life. The pitch parameter has no natural connection to distance and the design team defined low pitch as the distance "far", medium pitch as the distance "middle" and high pitch as "near" distance.

The first research question could be answered by analyzing how the test subjects agreed on the hypotheses defined by the test team. The second research question could be answered by analyzing which sound parameter or combination of parameters that gave a majority of test subjects the best sense of variation in distance.

In a pre-test, sound examples were created by modulating a basic sound from a vibraphone using the candidate sound parameters and all combinations of two and three of them. The vibraphone was selected based on its relatively short sound with a relatively distinct on-set and a relatively short decay. The vibraphone also has enough overtones to respond to low-pass filtering. In the context of the mobile application for navigation it is crucial that the sounds have distinct on-sets to separate them from each other and that they are kept short in order to overlap as little as possible. We wanted a short decay since a shorter decay makes it easier to hear the reverb tail added to the sound. On the other hand, the decay must have enough length so that the users can perceive the difference in low-pass filtering.

14 sound examples were prepared using the parameters shown in table 2. Each example contained three sounds modulated using the parameter / parameter combination. These three sounds corresponded to the distances "near", "middle" and "far".

Table 2: Initial candidates for sound parameters and parameter combinations to use in the test.

\begin{tabular}{cl}
\hline$\#$ & Parameters and parameter combinations \\
\hline 1 & Intensity \\
2 & Pitch \\
3 & Reverb \\
4 & Low-pass filter \\
5 & Intensity + pitch \\
6 & Intensity + reverb \\
7 & Intensity + low-pass filter \\
8 & Pitch + reverb \\
9 & Pitch + low-pass filter \\
10 & Reverb + low-pass filter \\
11 & Intensity + pitch + reverb \\
12 & Intensity + pitch + low-pass filter \\
13 & Intensity + reverb + low-pass filter \\
14 & Pitch + reverb + low-pass filter \\
\hline
\end{tabular}

Using this number of parameters / parameter combinations would make the test very extensive and potentially too exhausting for the test subjects. The pre-test was therefore used to reduce the number of parameters and parameter combinations. Four members of the research group carried out the pre-test internally by listening to all the examples in a number of diverse environments, from quiet offices to noisy streets. The set of parameters and parameter combinations was then discussed within the group.

As shown very early by for example Gamble [8], the intensity property is very prominent for experiencing distance through sound. But, as noted by Devallez et al., "the volume [of a smartphone] can be manipulated by users, which might make the intensity cue unreliable for judging distance". Potentially it is very difficult for the average user to determine a useful volume setting in order to actually hear sounds with low intensity. The intensity parameter is also problematic in urban and other outdoor settings, since sounds with lower intensity could be masked by background noise. When judging distance, the intensity effect is also depending on how familiar the user is with the sound source as shown by for example Coleman [4]. In the project's context of a mobile application, the intensity parameter was, for the reasons above, deemed too problematic and it was decided not to use it in the test.

The pre-test also showed that in sound examples where the basic sound was modified using a combination of pitch and low-pass filter, it was not possible to hear any effect of the change in lowpass filter, especially in outdoor settings. Instead only the change in pitch was clearly perceivable. It was therefore decided to omit also the parameter combination pitch + low-pass filter. This resulted in the list of remaining parameters and parameter combinations to use in the test shown in table 3.

Table 3. Sound parameters and parameter combinations selected for the test.

\begin{tabular}{ll}
\hline$\#$ & Parameter / parameter combination \\
\hline 1 & Pitch \\
2 & Reverb \\
3 & Low-pass filter \\
4 & Pitch + reverb \\
5 & Reverb + low-pass filter \\
\hline
\end{tabular}

\section{THE ACTUAL TEST}

The test was designed to assess how well the five remaining sound parameters and parameter combinations could be utilized to convey a sense of geographic distance.

All sounds originated from one basic, $300 \mathrm{~ms}$ long sound from a vibraphone at the pitch of D3 $(293.67 \mathrm{~Hz})$. Since sound timbre was not one of the parameters to test, all the sound examples used this same basic sound.

The basic sound from the vibraphone was then modified using the parameters and parameter combinations appointed for the test. Table 4 shows how the parameters/parameter combinations were used to modify the basic sound. Figure 1 shows the tones used for "near", "middle" and "far" when modifying the basic sound using the pitch parameter. The pre-test indicated that some people had difficulties hearing any difference between individual notes if the difference between those where too small. The relatively large difference in pitch is an attempt to make it possible for all to perceive this difference. 


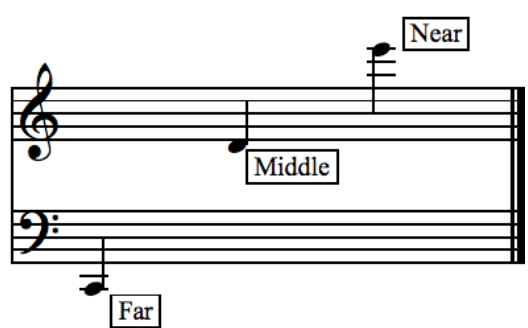

Figure 1. Tones used for distances "near", "middle" and "far".

The low-pass filter used in the test was the Steinberg Cubase's internal EQ. For the middle distance, the filter's cut-off frequency was set to $980 \mathrm{~Hz}$ with a Q-value of 7.0. For the far distance, the filter's cut-off frequency was set to $186 \mathrm{~Hz}$ with a Q-value of 5.7. For the near distance, the basic sound was unfiltered. The filter settings where made from listening and finding settings the test leaders perceived as relevant for the context of the test. The lowpass filtering changes the perceived intensity of the sound. Therefore, the volume of the filtered sounds where increased to compensate for the filtering.

The reverb effect used was Steinberg Cubase internal reverb "Roomworks". For all distances, the reverb parameters were kept constant with a reverb time of 1.7 seconds. Instead the send parameter from the basic sound was modified. For the middle distance, the send parameter was set to $-14,8 \mathrm{~dB}$. For the far distance, the send parameter was set to $0.0 \mathrm{~dB}$. For the near distance, the send parameter was muted. The reason for keeping the reverb time constant was to mimic the way reverberation works ecologically. The more distant a sound source is, the more reflected sound waves and less sound waves directly from the sound source will reach your ears. In the same way, the closer a sound source is to you, the more sound waves coming directly from the sound source you will hear and less reflected sound waves. Changing the send parameter to the reverb unit reflects this behavior by altering the balance between direct sound and reverberant sound.

Table 4. Sound modification methods used in the test.

\begin{tabular}{|c|c|c|c|c|c|}
\hline $\begin{array}{l}\text { Method/ } \\
\text { Distance }\end{array}$ & Pitch & $\begin{array}{l}\text { Low- } \\
\text { pass } \\
\text { filter }\end{array}$ & Reverb & $\begin{array}{l}\text { Pitch + } \\
\text { Reverb }\end{array}$ & $\begin{array}{l}\text { Low- } \\
\text { pass } \\
\text { Reverb }\end{array}+$ \\
\hline Near & High & None & None & $\begin{array}{l}\text { High } \\
\text { pitch, } \\
\text { no } \\
\text { reverb }\end{array}$ & $\begin{array}{l}\text { No filter, } \\
\text { no reverb }\end{array}$ \\
\hline Middle & Middle & Some & Some & $\begin{array}{l}\text { Middle } \\
\text { pitch, } \\
\text { some } \\
\text { reverb }\end{array}$ & $\begin{array}{l}\text { Some } \\
\text { filter, } \\
\text { some } \\
\text { reverb }\end{array}$ \\
\hline Far & Low & Heavy & Much & $\begin{array}{l}\text { Low } \\
\text { pitch, } \\
\text { much } \\
\text { reverb }\end{array}$ & $\begin{array}{l}\text { Heavy } \\
\text { filtering, } \\
\text { much } \\
\text { reverb }\end{array}$ \\
\hline
\end{tabular}

When the parameters were combined in pitch + reverb and lowpass filter + reverb, the individual parameter settings used were the same as when the parameters were used individually.

The test was divided in two parts and a total of 24 subjects were enrolled to do it. In the test's first part, the subjects listened to five examples, each consisting of three sounds. The task was to listen to the three sounds and for each example mark in a protocol which sound, 1, 2 and 3 they interpreted as the distances "near", "middle" and "far". The subjects were forced to do an exclusive choice, each distance had exactly one sound and each sound had exactly one distance. This part of the test gave a measurement on how much the subject agreed on the hypotheses and how intuitively interpretable and understandable each sound parameter/parameter combination is. The three sounds of each example were made by modifying the basic sound using one of the parameters / parameter combinations described above. The order of the examples and the relationship between the sounds 1,2 and 3 and the corresponding distances were randomized. The examples were presented to the test subjects using videos embedded in Apple Keynote presentations. Each sound was played synchronized with the digits 1,2 and 3 appearing visually in the video (see figure 2).

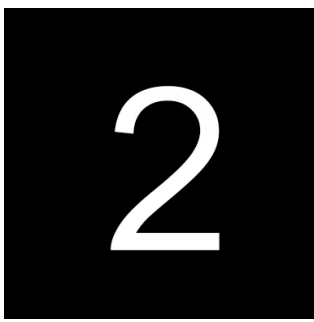

Figure 2. Screen shot of video from the test's first part.

The second part of the test was a use scenario reflecting the urban use of the sound-based mobile application for navigation. In this part of the test, the subjects compared alternative sound feedback solutions from the application. The alternative solutions were presented to the subjects as "mock-up videos" (figure 3).

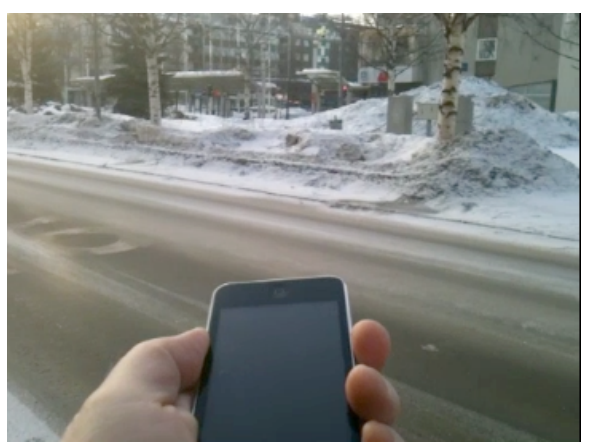

Figure 3. Screen shot of video from the test's second part.

Five different videos were prepared. Each video showed the same film sequence of a use scenario together with a background sound track with natural street sounds. Each video then had a second, unique sound track playing a number of "near", "middle" and "far" sounds in random order. In each video those sounds were composed of sounds modified using one of the test's parameters / 
parameter combination and based on the corresponding hypotheses.

Ten "rounds" with three different videos in each were prepared. In each "round", the test subjects listened to, watched and compared the three videos. The task was to select the video whose sound the test subject perceived was giving the best sense of difference between the distances "near", "middle" and "far". When all the ten "rounds" were finalized, the test subjects had compared all the five videos to all the rest. This was to get an indication on which of the parameters/parameter combinations the subjects perceived as giving the best sense of difference in distance. As in the first part of the test, the order in which the videos were presented was randomized.

In both parts of the test, the subjects could experience the examples as many times as they liked. They were also allowed to go back and revisit previous examples before making a final decision to put in the test protocol.

\section{RESULTS FROM PART 1}

In the first part of the test, subjects interpreted sounds in terms of "near", "middle" and "far". The sounds were modulated using the parameters and parameter combinations selected for the test. The subjects' interpretations of the sounds were compared to a set of hypotheses based on acoustics and previous work described above. The results from this part of the test show that test subjects agree most on the hypotheses for examples including the low-pass filter (see figure 4).

For examples based on the low-pass filter alone, $88 \%$ of the test subjects made interpretations in full agreement with the hypothesis. The same figure is true for examples based on the combination of low-pass filter and reverb. When interpreting examples based on the reverb parameter alone, $79 \%$ of the test subjects made interpretations in full agreement with the corresponding hypothesis. For the pitch parameter, the corresponding figure is $46 \%$. Examples based on the combination of pitch and reverb yields a better agreement with the hypothesis compared to examples based on pitch alone. For examples based on the combination of pitch and reverb, $67 \%$ of the test subjects made interpretations in full agreement with the hypothesis.

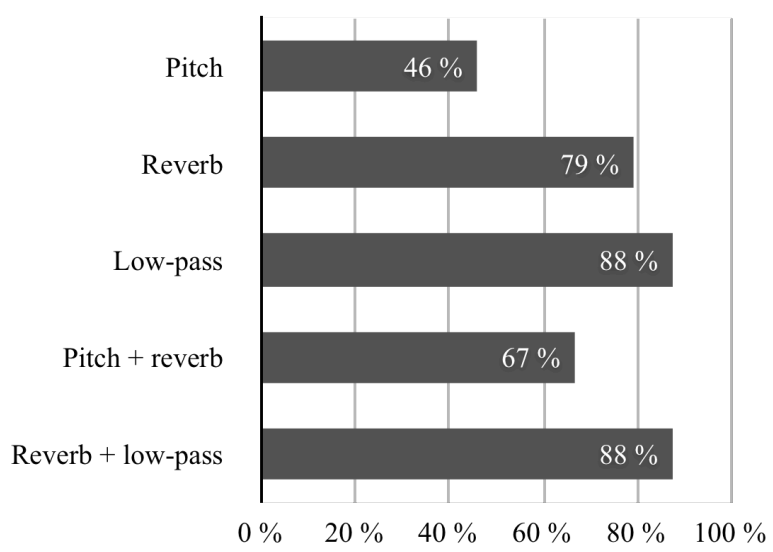

Figure 4. Percentage of test subjects in full agreement with the hypothesis for the parameters / parameter combinations
Figure 5 shows that $29 \%$ of the test subjects made interpretations that fully correspond to the hypotheses for all five examples. $33 \%$ of the subjects made interpretations that deviated from the hypotheses in one example. $21 \%$ of the subjects showed deviations in $2,8 \%$ in 3 and $8 \%$ in 4 examples. Each example corresponds to one parameter or parameter combination.

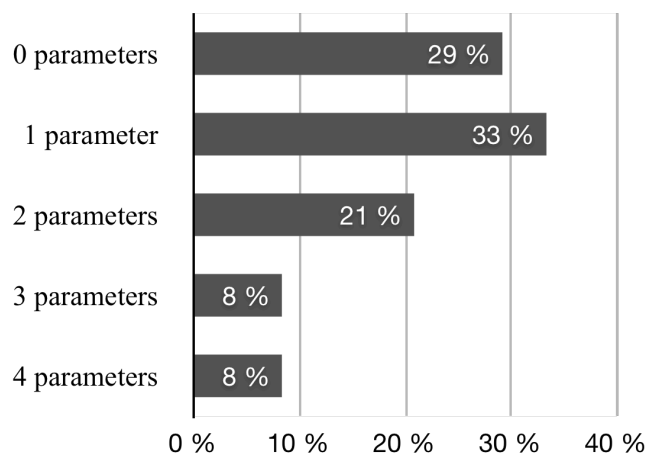

Figure 5. Percentage of test subjects with deviations in interpretations compared to the hypothesis in $0,1,2,3$ and 4 parameters/parameter combinations.

In total $20(83 \%)$ of the subjects made interpretations that deviate from the hypotheses in 0,1 or 2 examples. Of these 20 , one subject deviated in the low-pass filter example and one subject deviated in the reverb + low-pass filter example. Once again this indicates the low-pass filters ability to convey a sense of geographic distance.

\section{RESULTS FROM PART 2}

In the second part of the test, subjects compared five sound design solutions based on the five acoustic parameters and parameter combinations used in the test. With 24 subjects, each parameter/ parameter combination could be the preferred one in total 144 times.

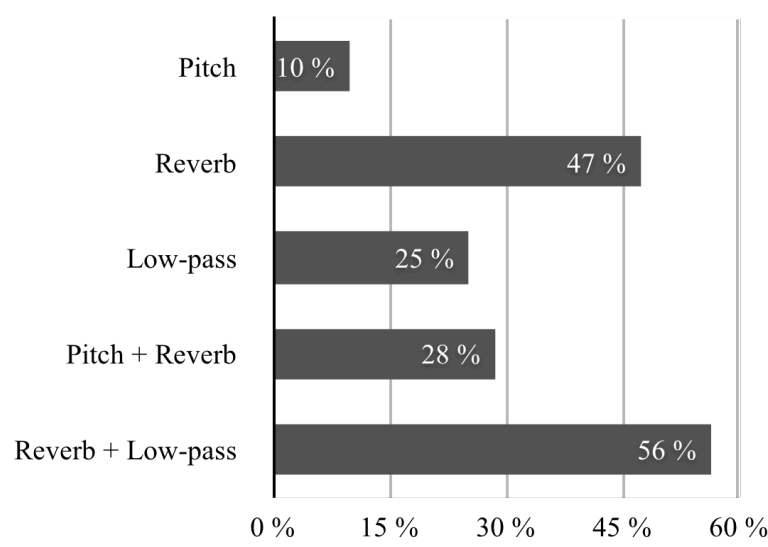

Figure 6. Preferences for parameters and parameter combinations with respect to ability to convey difference in geographic distance.

The results from this part of the test show that the solution based on the parameter combination reverb + low-pass filter was preferred most times, in $56 \%$ of the possible comparisons (81 of 
maximum 144 times, see figure 6). The corresponding figure for the solution based on reverb is $47 \%$, pitch + reverb $28 \%$, low-pass filter $25 \%$ and for solutions based on the pitch parameter the figure is $10 \%$.

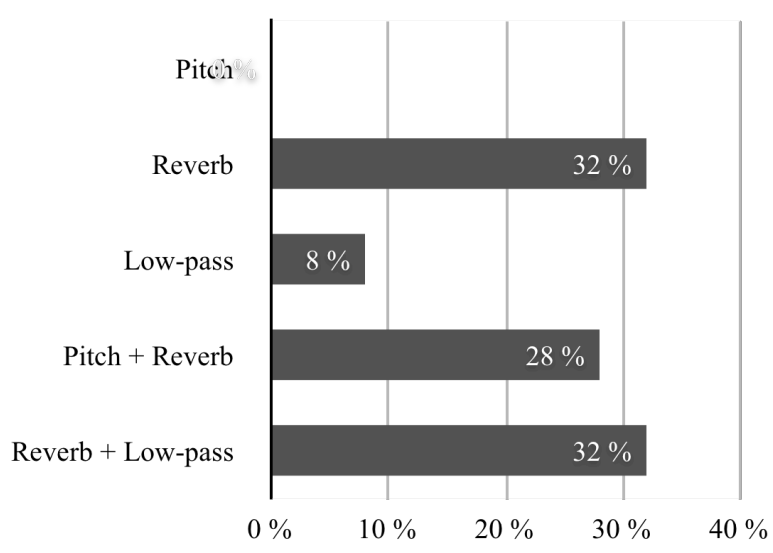

Figure 7. Percentage of subjects preferring each parameter / parameter combination.

Looking at the same comparisons from a per subject perspective, $32 \%$ of the subjects selected the solution based on the reverb + low-pass filter in most comparisons (see figure 7). Another $32 \%$ of the subjects in most comparisons selected the solution built on the reverb parameter. For pitch + reverb the figure is $28 \%$ and for the low-pass filter the figure is $8 \%$. No test subject selected pitch in most of the comparisons.

\section{DISCUSSION}

In the test, the subjects considered the proposed solutions without any prior information or knowledge about how the design team intended the solutions to be interpreted. Instead the subjects were asked to describe, without being biased, their interpretations of the solutions and express their preferences between those. The results from the first part of the test indicate that the reverb and the lowpass filter parameters were the ones the subjects interpreted most in line with the corresponding hypothesis. The interpretations of the solutions including the pitch parameter were the ones that deviated most from the design team's hypothesis. This is in line with previous work. It is interesting to note that the results are consistent also with the findings from Talbot and Cowan's tests that used visually impaired subjects.

Of the parameters selected for and used in the test, the reverb and the low-pass filter parameters can be described as the ones that are most in line with our everyday experiences of how sound works on various distances. Sound sources far away sound more dull or damped than sounds from sound sources closer to the listener. Sound from far away sound sources are often reverberant compared to sound from closer sound sources. This is the way we are used to perceive sound in our everyday lives. On the other hand, there is no immediate and intuitive relationship between a certain pitch and the concepts of "near", "middle" and "far". It is therefore not surprising that the test subjects' deviated more from the design team's hypotheses when interpreting solutions that included the pitch parameter compared to solutions based on the reverb and low-pass filter parameters. This is also in line with previous findings.
Part two of the test exhibits a similar pattern. Figure 7 shows that $92 \%$ of the test subjects preferred examples based on the reverb parameter or combinations including the reverb parameter. Reverb seems to be an acoustic parameter that is a strong carrier of information about geographic distance.

The picture is somewhat complicated by the low-pass filter parameter. The results from the test's part 1 show that the examples including the low-pass filter parameter are the examples for which the test subject's interpretations deviated the least from the design team's ecologically based and motivated hypothesis. On the other hand, in part 2 of the test, examples modulated with the low-pass filter alone are most often not preferred by the test subjects. The subjects' conscious preferences and their ability to interpret stimuli based on the low-pass filter parameter seems to differ.

A possible explanation to this difference is that part1 of the test concentrated on the interpretation of sound parameters in terms of the distances "near", "middle" and "far" in relative isolation from any use scenario or other context. In part 1 the subjects could concentrate fully on the interpretation of the sounds alone. The use case scenario in part 2 might have distracted the subjects somewhat. Part 2 deals with the question which sound parameter / parameter combination best convey a sense of "near", "middle" and "far" when the sounds are put in a use scenario. The low-pass filter seems to be able to convey relatively unambiguous information about "near", "middle" and "far" when heard in relative isolation. When heard as part of a larger context, the lowpass filter alone might sound less differentiated compared to sounds modulated with other parameters. Informal statements by some of the test subjects also support this possible explanation.

The test concentrated on the test subject's immediate and intuitive responses and interpretations of five acoustic parameters and parameter combinations. Any longer term learning aspects of repeated encounters with the parameters / parameter combinations were not included in the test or the analysis of the results.

The results from the test suggest using combinations of two or more acoustic parameters when distance and difference in distances are to be conveyed. This idea is corroborated by other findings.

Reverberation is a strong carrier of distance information. But this parameter is dependant on a sound's development over time. If the time span for each sound is narrow and/or many sounds are played in close succession or even overlapping, the risk is that the reverb parameter will not be heard and therefore the perception of distance lost. Once again, combining parameters might help reduce the problem.

\section{FUTURE WORK AND USE OF THE RESULTS}

The results from this experiment will be fed into the development process of a multi-modal, mobile application for navigation. Based on the results reported above, this application will have a sound design that uses combinations of several acoustic parameters to convey information about distance. The application will be evaluated using both qualitative and quantitative methods in order to assess users' experience of the application, potential meanings it can be given and roles it may play in people's lives.

Most likely, based on the results and experiences from the experiment reported here, the sound design team will use a combination of low-pass filtering, reverberation, sound intensity and pitch to convey the difference between "near", "middle" and 
"far". Since the sound intensity parameter has to be handled with caution, it will be used only to a small, just noticeable degree. The pitch parameter will be used to distinguish sounds played on close succession. The pitch span used will be radically smaller than the span used in this test, probably within the interval of a quint.

\section{ACKNOWLEDGMENTS}

We thank all our subjects who volunteered for the test. We also thank our colleagues, Katarina Delsing, Johan Fagerlönn and Nigel Papworth for invaluable input and ideas for the test design.

\section{REFERENCES}

[1] Amemiya, T., Ando, H. and Maeda, T. Lead-me interface for a pulling sensation from hand-held devices. ACM Trans. Appl. Percept. 5, 3, Article 15 2008, pp. 1-17.

[2] Bregman, A.S. Auditory scene analysis: the perceptual organization of sound. MIT Press, Cambridge, MA, USA, 1990.

[3] Brungart, D. Auditory localization of nearby sources III: Stimulus effects. Journal of Acoustical Soc. Am., 106:35893602, 1999.

[4] Coleman, P.D. An Analysis of Cues to Auditory Depth Perception in Free Space. Psychological Bulletin, 1963, Vol. 60 , No. 3, 302-315.

[5] Devallez, D., Rocchesso, D. and Fontana, F. An AudioHaptic Interface Concept Based on Depth Information. In Proc. HAID 2008, Springer-Verlag, $102-110$.

[6] Djajadiningrat, T., Wensveen, S., Frens, J., and Overbeeke, $\mathrm{K}$. Tangible products: redressing the balance between appearance and action. Personal and Ubiquitous Computing 8. Springer-Verlag, London, 294-309.

[7] Everest, F. Alton. The Master Handbook of Acoustics. Fourth edition. MacGraw-Hill, New York, NY, 2001

[8] Gamble, E.A. Intensity as a criterion in estimating the distance of sounds. In Psychol. Rev., 16:416-426, 1909.

[9] Google Street View. http://maps.google.com/intl/en_us/help/maps/streetview

[10] Holland, S., Morse, D.R., and Gedenryd, H. AudioGPS: spatial audio navigation with a minimal attention interface. Personal Ubiquitous Computing 6(4), 253-259.

[11] Jones, M., Jones, S., Bradley, G., Warren, N., Bainbridge, D., and Holmes, G. 2008. Ontrack: dynamically adapting music playback to support navigation. Personal and Ubiquitous Computing, 12. Springer-Verlag, London, 513-525.
[12] Kragh, J., Plovsing, B., Storeheier, S. Å., Taraldsen, G. and Jonasson, H. G. Nordic Environmental Noise Prediction Methods, Nord2000. Summary Report. General Nordic Sound Propagation Model and Applications in SourceRelated Prediction Methods. Lyngby, Denmark: DELTA Acoustics \& Vibration.

[13] MacEachren, A. M. How maps work: representation, visualization, and design. The Guilford Press, 1995.

[14] McGookin, D., Brewster, S., and Priego, P. Audio Bubbles: Employing Non-speech Audio to Support Tourist Wayfinding. In Proc. HAID 2009, Springer-Verlag, 41 - 50.

[15] O'Callaghan, C. Auditory perception. In E. N. Zalta (Ed.), The Stanford encyclopedia of philosophy, Retrieved February 16, 2011, from http://www.seop.leeds.ac.uk/entries/perception-auditory/.

[16] Martin Talbot and William Cowan. 2009. On the audio representation of distance for blind users. In Proceedings of the 27th international conference on Human factors in computing systems (CHI '09). ACM, New York, NY, USA, 1839-1848. DOI=10.1145/1518701.1518984 http://doi.acm.org/10.1145/1518701.1518984

[17] Martin Pielot, Niels Henze, and Susanne Boll. 2009. Supporting map-based wayfinding with tactile cues. In Proceedings of the 11th International Conference on HumanComputer Interaction with Mobile Devices and Services (MobileHCI '09). ACM, New York, NY, USA, , Article 23 , 10 pages. $\mathrm{DOI}=10.1145 / 1613858.1613888$ http://doi.acm.org/10.1145/1613858.1613888

[18] Robinson, S., Jones, M., Eslambolchilar, P., Murray-Smith, R. and Lindborg, M. "I did it my way": moving away from the tyranny of turn-by-turn pedestrian navigation. In Proc. of MobileHCI '10. ACM, 341-344.

[19] Spath, D., Peissner, M., Hagenmeyer, L. and Ringbauer, B. New Approaches to Intuitive Auditory User Interfaces. In M.J. Smith, G. Salvendy (Eds.): Human Interface, Part I, HCII 2007, LNCS 4557, pp. 975-984, 2007.

[20] Tractinsky, N. Aesthetics and apparent usability: empirically assessing cultural and methodological issues. In Proc. Of CHI '97. 115-122, ACM.

[21] Tsukada, K. and Yasymua, M. ActiveBelt: Belt-Type Wearable Tactile Display for Directional Navigation. Proc. of Ubicomp 04, Springer, pp. 384-399.

[22] Wu, A., Zhang, W. and Zhang X. Evaluation of Wayfinding Aids in Virtual Environment. In Intl. Journal of HumanComputer Interaction 25(1), 1-21, 2009. 